\title{
Distances between nested densities and a measure of the impact of the prior in Bayesian statistics
}

\author{
Christophe Ley*, Gesine Reinert ${ }^{\dagger}$ and Yvik Swan ${ }^{\ddagger}$ \\ Christophe Ley \\ Ghent University \\ Department of Applied Mathematics, \\ Computer Science and Statistics \\ Krijgslaan 281, S9 \\ 9000 Ghent, Belgium \\ e-mail: christophe.ley@ugent.be \\ Gesine Reinert \\ University of Oxford \\ Department of Statistics \\ 1 South Parks Road \\ Oxford OX1 3TG, UK \\ e-mail: reinert@stats.ox.ac.uk \\ Yvik Swan \\ Université de Liège \\ Département de Mathématique \\ 12 allée de la découverte \\ Bât. B37 pkg $33 a$ \\ 4000 Liège, Belgium \\ e-mail: yswan@ulg.ac.be
}

\begin{abstract}
In this paper we propose tight upper and lower bounds for the Wasserstein distance between any two univariate continuous distributions with probability densities $p_{1}$ and $p_{2}$ having nested supports. These explicit bounds are expressed in terms of the derivative of the likelihood ratio $p_{1} / p_{2}$ as well as the Stein kernel $\tau_{1}$ of $p_{1}$. The method of proof relies on a new variant of Stein's method which manipulates Stein operators.

We give several applications of these bounds. Our main application is in Bayesian statistics : we derive explicit data-driven bounds on the Wasserstein distance between the posterior distribution based on a given prior and the no-prior posterior based uniquely on the sampling distribution. This is the first finite sample result confirming the well-known fact that with well-identified parameters and large sample sizes, reasonable choices of prior distributions will have only minor effects on posterior inferences if the data are benign.
\end{abstract}

Keywords and phrases: Stein's method, Bayesian analysis, Prior distribution, Posterior distribution.

\section{Introduction}

A key question in Bayesian analysis is the effect of the prior on the posterior, and how this effect could be assessed. As more and more data are collected, will the posterior distributions derived with different priors be very similar? This question has a long history; see for example $([26,4,3])$. While asymptotic results which give conditions under which the effect of the prior wanes as the sample size tends to infinity can be found for example in $[4,3]$, here we are interested, at fixed sample size, in explicit bounds on some measure of the distributional distance between posteriors based on a given prior and the no-prior data-only based posterior, allowing to detect at fixed sample size the effect of the prior.

\footnotetext{
${ }^{*}$ Christophe Ley, whose affiliation during parts of this work was Université libre de Bruxelles, thanks the Fonds National de la Recherche Scientifique, Communauté française de Belgique, for financial support via a Mandat de Chargé de Recherche FNRS.

$\dagger$ Gesine Reinert acknowledges support from EPSRC grant EP/K032402/I as well as from the Keble College Advanced Studies Centre.

${ }^{\ddagger}$ Yvik Swan gratefully acknowledges support from the IAP Research Network P7/06 of the Belgian State (Belgian Science Policy).
} 
In the simple setting of prior and posterior being univariate and continuous, the basic relation that the posterior is proportional to the prior times the likelihood leads to the more general problem of comparing two distributions $P_{1}$ and $P_{2}$ whose densities $p_{1}$ and $p_{2}$ have nested supports. Letting $\mathcal{I}_{1}$ (resp., $\mathcal{I}_{2}$ ) be the support of $p_{1}$ (resp., $p_{2}$ ) and assuming $\mathcal{I}_{2} \subset \mathcal{I}_{1}$ we can write

$$
p_{2}=\pi_{0} p_{1}
$$

for $\pi_{0}=p_{2} / p_{1}$ a non-negative finite function called likelihood ratio in statistics. To assess the distance between such distributions, we choose the Wasserstein-1 distance defined as

$$
d_{\mathcal{W}}\left(P_{1}, P_{2}\right)=\sup _{h \in \mathcal{H}}\left|\mathbb{E}\left[h\left(X_{2}\right)\right]-\mathbb{E}\left[h\left(X_{1}\right)\right]\right|
$$

for $\mathcal{H}=\operatorname{Lip}(1)$ the class of Lipschitz-1 functions, where $X_{1}$ has distribution $P_{1}$ (resp., probability distribution function (pdf) $p_{1}$ ) and $X_{2}$ has distribution $P_{2}$ (resp., pdf $p_{2}$ ). The central aim of this paper is to provide meaningful bounds on $d_{\mathcal{W}}\left(P_{1}, P_{2}\right)$ in terms of $\pi_{0}$.

Our approach to this problem relies on Stein's density approach introduced in [27, 28], as further developed in $[15,16,17,18]$. Let $P_{1}$ have density $p_{1}$ with interval support $\mathcal{I}_{1}$ with closure $\left[a_{1}, b_{1}\right]$ for some $-\infty \leq a_{1}<b_{1} \leq+\infty$. Suppose also that $P_{1}$ has mean $\mu$. Then a notion which will be of particular importance is the Stein kernel of $P_{1}$ which is the function $\tau_{1}:\left[a_{1}, b_{1}\right] \rightarrow \mathbb{R}$ given by

$$
\tau_{1}(x)=\frac{1}{p_{1}(x)} \int_{a_{1}}^{x}(\mu-y) p_{1}(y) d y .
$$

Our main results assume that $p_{1}$ and $p_{2}$ are absolutely continuous densities, and that $\pi_{0}$ is a differentiable function satisfying

Assumption A : $\lim _{x \rightarrow a_{1}} \pi_{0}(x) \int_{a_{1}}^{x}\left(h(y)-\mathbb{E}\left[h\left(X_{1}\right)\right]\right) p_{1}(y) d y=0=\lim _{x \rightarrow b_{1}} \pi_{0}(x) \int_{x}^{b_{1}}\left(h(y)-\mathbb{E}\left[h\left(X_{1}\right)\right]\right) p_{1}(y) d y$ for all Lipschitz-continuous functions $h$ with $\left|\mathbb{E}\left[h\left(X_{1}\right)\right]\right|<\infty$. Here $X_{1} \sim P_{1}$.

Under these assumptions we prove the following result (Theorem 3.1).

Theorem. The Wasserstein distance between $P_{1}$ with pdf $p_{1}$ and $P_{2}$ with $p d f p_{2}=\pi_{0} p_{1}$ satisfies the following inequalities:

$$
\left|\mathbb{E}\left[\pi_{0}^{\prime}\left(X_{1}\right) \tau_{1}\left(X_{1}\right)\right]\right| \leq d_{\mathcal{W}}\left(P_{1}, P_{2}\right) \leq \mathbb{E}\left[\left|\pi_{0}^{\prime}\left(X_{1}\right)\right| \tau_{1}\left(X_{1}\right)\right]
$$

where $\tau_{1}$ is the Stein kernel associated with $p_{1}$ and $X_{1} \sim P_{1}$.

If $P_{1}=\mathcal{N}\left(\mu, \sigma^{2}\right)$ is a normal distribution then the above result simplifies considerably because $\tau_{1}(x)=\sigma^{2}$ is constant, yielding

$$
\sigma^{2}\left|\mathbb{E}\left[\pi_{0}^{\prime}\left(X_{1}\right)\right]\right| \leq d_{\mathcal{W}}\left(P_{1}, P_{2}\right) \leq \sigma^{2} \mathbb{E}\left[\left|\pi_{0}^{\prime}\left(X_{1}\right)\right|\right] .
$$

The Gaussian is characterized by the fact that its Stein kernel is constant. More generally, all distributions belonging to the classical Pearson family possess a polynomial Stein kernel (see [27]). The problem of determining the Stein kernel is, in general, difficult. Even when the Stein kernel $\tau_{1}$ is not available we can give the following simpler bound (Corollary 3.5).

Corollary. Under the same assumptions as in Theorem 3.1,

$$
\left|\mathbb{E}\left[X_{1}\right]-\mathbb{E}\left[X_{2}\right]\right| \leq d_{\mathcal{W}}\left(P_{1}, P_{2}\right) \leq\left\|\pi_{0}^{\prime}\right\|_{\infty} \operatorname{Var}\left[X_{1}\right] .
$$

More generally, because the Stein kernel is always positive, the upper and lower bounds in the Theorem turn out to be the same whenever the likelihood ratio $\pi_{0}$ is monotone, which is equivalent 
to requiring that $P_{1}$ and $P_{2}$ are stochastically ordered in the sense of likelihood ratios. This brings our next result (Corollary 3.6).

Corollary. Let $X_{1} \sim P_{1}$ and $X_{2} \sim P_{2}$. If $X_{1} \leq_{L R} X_{2}$ or $X_{2} \leq_{L R} X_{1}$ then

$$
d_{\mathcal{W}}\left(P_{1}, P_{2}\right)=\left|\mathbb{E}\left[X_{2}\right]-\mathbb{E}\left[X_{1}\right]\right|=\mathbb{E}\left[\left|\pi_{0}^{\prime}\left(X_{1}\right)\right| \tau_{1}\left(X_{1}\right)\right]=\mathbb{E}\left[\left|\left(\log \pi_{0}\left(X_{2}\right)\right)^{\prime}\right| \tau_{1}\left(X_{2}\right)\right] .
$$

In case of a monotone likelihood ratio between $P_{1}$ and $P_{2}$, the first of the above identities is easy to derive directly from the known alternative definitions of the Wasserstein distance (see e.g. [29])

$$
d_{\mathcal{W}}\left(P_{1}, P_{2}\right)=\int_{-\infty}^{\infty}\left|F_{P_{1}}(x)-F_{P_{2}}(x)\right| d x=\int_{0}^{1}\left|F_{P_{1}}^{-1}(u)-F_{P_{2}}^{-1}(u)\right| d u
$$

with $F_{P_{1}}$ and $F_{P_{1}}^{-1}$ (resp., $F_{P_{2}}$ and $F_{P_{2}}^{-1}$ ) the cumulative distribution function and quantile function of $P_{1}$ (resp., $P_{2}$ ).

We illustrate the effectiveness of our bounds in several examples at the end of Section 3.1, comparing e.g. Gaussian random variables or Azzalini's skew-symmetric densities with their symmetric counterparts. In Section 4 we treat as main application the Bayes example wherein we measure explicitly the effect of priors on posterior distributions. Suppose we observe data points $x:=\left(x_{1}, x_{2}, \ldots, x_{n}\right)$ with sampling density $f(x ; \theta)$ (proportional to the likelihood), where $\theta$ is the one-dimensional parameter of interest. Let $p_{0}(\theta)$ be a certain prior distribution, possibly improper, and let $\Theta_{2}$ be the resulting posterior guess for $\theta$ perceived as a random variable. By Bayes' theorem, this has density $p_{2}(\theta ; x)=\kappa_{2}(x) f(x ; \theta) p_{0}(\theta)$ with $\kappa_{2}(x)$ the normalizing constant which depends on the data. Under moderate assumptions, we provide computable expressions for the Wasserstein distance $d_{\mathcal{W}}\left(\Theta_{2}, \Theta_{1}\right)$ between this posterior distribution and $\Theta_{1}$, whose law is the no-prior posterior distribution with density (proportional to the likelihood) given by $p_{1}(\theta ; x)=\kappa_{1}(x) f(x ; \theta)$, again with normalizing constant $\kappa_{1}(x)$ depending on the data. The bounds we derive are expressed in terms of the data, the prior and the Stein kernel $\tau_{1}$ of the sampling distribution.

We study the normal model with general and normal priors, the binomial model under a general prior, a conjugate prior, and the Jeffreys' prior. We also consider the Poisson model with an exponential prior, in which case we can make use of the likelihood ratio ordering. For example, with a normal $\mathcal{N}\left(\mu, \delta^{2}\right)$ prior and a random sample $x_{1}, \ldots, x_{n}$ from a normal $\mathcal{N}\left(\theta, \sigma^{2}\right)$ model with fixed $\sigma^{2}$, we obtain in (4.4) that

$$
\frac{\sigma^{2}}{n \delta^{2}+\sigma^{2}}|\bar{x}-\mu| \leq d_{\mathcal{W}}\left(\Theta_{1}, \Theta_{2}\right) \leq \frac{\sigma^{2}}{n \delta^{2}+\sigma^{2}}|\bar{x}-\mu|+\frac{\sqrt{2}}{\sqrt{\pi}} \frac{\sigma^{3}}{n \delta \sqrt{\delta^{2} n+\sigma^{2}}} .
$$

Not only do we see that for $n \rightarrow \infty$, the distance becomes zero, as is well known, but we also have an explicit dependence on the difference between the sample mean $\bar{x}$ and the prior mean $\mu$, indicating the importance of a reasonable choice for the prior. For a normal $\mathcal{N}\left(\theta, \sigma^{2}\right)$ model and a general prior on $\theta$, we obtain in (4.3) that

$$
\frac{\sigma^{2}}{n}\left|\mathbb{E}\left[\rho_{0}\left(\Theta_{2}\right)\right]\right| \leq d_{\mathcal{W}}\left(\Theta_{1}, \Theta_{2}\right) \leq \frac{\sigma^{2}}{n} \mathbb{E}\left[\left|\rho_{0}\left(\Theta_{2}\right)\right|\right]
$$

with $\rho_{0}$ the score function of the prior distribution. Here the data are hidden in the distribution of $\Theta_{2}$. In the binomial case with conjugate prior we obtain

$$
\begin{array}{r}
\frac{1}{n+2}\left|(2-\alpha-\beta) \frac{\frac{\alpha}{n}+\bar{x}}{1+\frac{\alpha+\beta}{n}}+(\alpha-1)\right| \leq d_{\mathcal{W}}\left(\Theta_{1}, \Theta_{2}\right) \\
\leq \frac{1}{n+2}\left(|2-\beta-\alpha| \frac{\frac{\alpha}{n}+\bar{x}}{1+\frac{\alpha+\beta}{n}}+|\alpha-1|\right),
\end{array}
$$


with $\alpha$ and $\beta$ the parameters of the conjugate (beta) prior. Finally in the Poisson case we obtain

$$
d_{\mathcal{W}}\left(\Theta_{1}, \Theta_{2}\right)=\frac{\lambda}{n+\lambda} \bar{x}+\frac{\lambda}{n(n+\lambda)} .
$$

with $\lambda>0$ the parameter of the exponential prior.

The main tool in this paper is a specification of the general approach in [15] which allows to manipulate Stein operators. Distributions can be compared through their Stein operators which are far from being unique; for a single distribution there is a whole family of operators which could serve as Stein operators, see for example [15]. In this paper, for probability distribution $P$ with pdf $p$ we choose the Stein operator $\mathcal{T}_{P}$ as

$$
\mathcal{T}_{P}: f \mapsto \mathcal{T}_{P} f=\frac{(f p)^{\prime}}{p}
$$

with the convention that $\mathcal{T}_{P} f(x)=0$ outside of the support of $P$; for details see Definition 2.1 and [18]. For this choice of operator, the product structure implies a convenient connection between $\mathcal{T}_{1}$, the Stein operator for $P_{1}$ with pdf $p_{1}$, and $\mathcal{T}_{2}$, the Stein operator for $P_{2}$ with pdf $p_{2}=\pi_{0} p_{1}$, namely

$$
\mathcal{T}_{2}(f)=\mathcal{T}_{1}(f)+f \frac{\pi_{0}^{\prime}}{\pi_{0}}=\mathcal{T}_{1}(f)+f\left(\log \pi_{0}\right)^{\prime} ;
$$

see (3.2). The difference

$$
\mathcal{T}_{2}(f)-\mathcal{T}_{1}(f)=f\left(\log \pi_{0}\right)^{\prime}
$$

is the cornerstone of our results.

Remark 1.1. This paper restricts attention to the univariate case. The multivariate case is of considerable interest but our approach requires an extension of the density method to a multivariate setting, which is to date still under construction and not yet available.

Using the approach in [15] it would be possible to extend our results to more general RadonNikodym derivatives, at the expense of clarity of exposition.

The paper is organized as follows. In Section 2, we provide the necessary notations and definitions from Stein's method, which allows us to state our main result, Theorem 3.1, in Section 3.1. Several applications of this result are discussed in Examples 3.3 to 3.9, while Section 4 tackles our motivating Bayesian problem by providing a measure of the impact of the choice of the prior on the posterior distribution for finite sample size $n$. Finally in Section 5 we provide a proof of one of the crucial bounds we need for our estimation purposes.

\section{A review of Stein's density approach}

\subsection{Notations and definitions}

Here we recall some notions from [15] and [18]. Consider a probability distribution $P$ with continuous univariate Lebesgue density $p$ on the real line and let $L^{1}(p)=L^{1}(p(x) d x)$ denote the collection of $f: \mathbb{R} \rightarrow \mathbb{R}$ such that $\mathbb{E}|f(X)|=\int|f(x)| p(x) d x<\infty$, where $X \sim P$. Let $\mathcal{I}=\{x \in \mathbb{R} \mid p(x)>0\}$ be the support of $p$. In this paper we shall use the following definition of a Stein operator; see for example [15] for a discussion of alternative choices.

Definition 2.1. [Stein pair] The Stein class $\mathcal{F}(P)$ of $P$ is the collection of $f: \mathbb{R} \rightarrow \mathbb{R}$ such that (i) $f p$ is absolutely continuous, (ii) $(f p)^{\prime} \in L^{1}(d x)$ and (iii) $\int_{\mathbb{R}}(f p)^{\prime} d x=0$. The Stein operator $\mathcal{T}_{P}$ for $P$ is

$$
\mathcal{T}_{P}: \mathcal{F}(P) \rightarrow L^{1}(p): f \mapsto \mathcal{T}_{P} f=\frac{(f p)^{\prime}}{p}
$$

with the convention that $\mathcal{T}_{P} f(x)=0$ outside of $\mathcal{I}$. 
Here $(f p)^{\prime}$ denotes the derivative of $f p$ which exists Lebesgue-almost surely due to the assumption of absolute continuity. Often the Stein pair $\left(\mathcal{F}(P), \mathcal{T}_{P}\right)$ is written as dependent on $X \sim P$ rather than on $P$ (that is, as $\left(\mathcal{F}(X), \mathcal{T}_{X}\right)$ ); we use the dependence on the distribution to emphasize that the pair itself is not random.

Note that because we only consider $f$ multiplied by $p$ the behavior of $f$ outside of $\mathcal{I}$ is irrelevant.

Remark 2.2. A sufficient condition for $\mathcal{F}(P) \neq \emptyset$ is that $p^{\prime}$ is integrable with integral 0 so that e.g. $f=1 \in \mathcal{F}(P)$. Such an assumption is in general too strong (see e.g. [28] for a discussion about the arcsine distribution) and weaker assumptions on $p$ are permitted in our framework, although in such cases stronger constraints on the functions in $\mathcal{F}(P)$ are necessary. In particular the constant functions may not belong to $\mathcal{F}(P)$.

All random quantities appearing in the sequel will be assumed to have non-empty Stein class (an assumption verified for all classical distributions from the literature).

It is easy to see from Definition 2.1 (iii) that $\mathbb{E}\left[\mathcal{T}_{P} f(X)\right]=0$ for all $f \in \mathcal{F}(P)$. More generally one can prove that if $Y$ and $X$ share the same support then $Y \stackrel{\mathcal{D}}{=} X$ (equality in distribution) if and only if $\mathbb{E}\left[\mathcal{T}_{P} f(Y)\right]=0$ for all $f \in \mathcal{F}(P)$. For any family of operators $\mathcal{T}$ indexed by univariate probability measures $P$ and $Q$ and for any class of functions $\mathcal{G}$ we say that $\left(\mathcal{T}_{P}, \mathcal{G}\right)$ is a Stein characterization if

$$
P=Q \Longleftrightarrow \mathcal{T}_{Q}(f)=\mathcal{T}_{P}(f) \quad \forall f \in \mathcal{G}
$$

see $[18,16]$ for general versions. In particular a Stein pair $\left(\mathcal{T}_{P}, \mathcal{F}(P)\right)$ is a Stein characterization.

With our notations, the operator $\mathcal{T}_{P}$ also admits an inverse which is easy to write out formally at least. Let $X \sim P$ have (open, closed, or half-open) interval support $\mathcal{I}$ between $a$ and $b$, where $-\infty \leq a<b \leq+\infty$ and

$$
\mathcal{F}^{(0)}(P)=\left\{h \in L^{1}(p): \mathbb{E}[h(X)]=0\right\} .
$$

Define $\mathcal{T}_{P}^{-1}: \mathcal{F}^{(0)}(P) \rightarrow \mathcal{F}(P)$ by

$$
\mathcal{T}_{P}^{-1} h(x)=\frac{1}{p(x)} \int_{a}^{x} h(y) p(y) d y=-\frac{1}{p(x)} \int_{x}^{b} h(y) p(y) d y .
$$

The operator $\mathcal{T}_{P}^{-1}$ is the inverse Stein operator of $P$ in the sense that

$$
\mathcal{T}_{P}\left(\mathcal{T}_{P}^{-1} h\right)=h .
$$

Note how the particular structure of the r.h.s. of (2.3) ensures that $\mathcal{T}_{P}^{-1} h$ belongs to $\mathcal{F}(P)$ for any $h \in \mathcal{F}^{(0)}(P)$. If in addition $(f p)(a)=(f p)(b)=0$ for all $f \in \mathcal{F}(p)$ then

$$
\mathcal{T}_{P}^{-1}\left(\mathcal{T}_{P} f\right)=f
$$

so that $\mathcal{T}_{P}^{-1}$ constitutes a bona fide inverse in this case.

\subsection{Standardizations of the operator}

Although the Stein pair $\left(\mathcal{T}_{P}, \mathcal{F}(P)\right)$ is uniquely defined in Definition 2.1, there are many implicit conditions on $f \in \mathcal{F}(P)$ which are useful to identify before applying this construction to specific approximation problems. In particular for favourable behavior of the inverse Stein operator it may be advantageous to consider only subclasses $\mathcal{F}_{\text {sub }}(P) \subset \mathcal{F}(P)$ of functions satisfying certain target-specific and well chosen constraints. A good choice of subclass will lead to specific forms of the resulting operator which may turn out to have a smooth inverse Stein operator, as illustrated in the next example. As long as $\mathcal{F}_{\text {sub }}(P)$ is a measure-determining class, the class is informative enough to satisfy (2.2). 
Example 2.3. In the case of the Laplace distribution Lap with $p d f p(x) \propto e^{-|x|}$ the Stein operator from Definition 2.1 is

$$
\mathcal{T}_{\text {Lap }} f(x)=f^{\prime}(x)-\operatorname{sign}(x) f(x)
$$

with $f \in \mathcal{F}(\mathrm{Lap})$, the class of functions such that $f(x) e^{-|x|}$ is differentiable almost surely with integrable derivative, and the derivative of $f(x) e^{-|x|}$ integrates to 0 over the real line. This operator does not have agreeable properties, mainly because the assumptions on $\mathcal{F}(\mathrm{Lap})$ are not explicit (see e.g. [10] and [22]). It is indeed sufficient to consider functions of the form $f(x)=\left(x f_{0}(x) e^{|x|}\right)^{\prime} / e^{|x|}$ for certain functions $f_{0}$. Applying $\mathcal{T}_{\text {Lap }}$ to such functions yields the second order operator

$$
\mathcal{T}_{\text {Lap }} f(x)=\mathcal{A}_{X} f_{0}(x)=x f_{0}^{\prime \prime}(x)+2 f_{0}^{\prime}(x)-x f_{0}(x)
$$

with $f_{0} \in \mathcal{F}\left(\mathcal{A}_{\text {Lap }}\right)$ the class of functions which are piecewise twice continuously differentiable such that $x f_{0}^{\prime \prime}(x), f_{0}^{\prime}(x)$ and $x f_{0}(x)$ are all in $L^{1}\left(e^{-|x|} d x\right)$, as considered e.g. in [10, 11]. In [22] functions of the form $f(x)=\left(-(g(x)-g(0)) e^{|x|}\right)^{\prime} / e^{|x|}$ yielded the second order operator

$$
\mathcal{T}_{\text {Lap }, P R} g(x)=g(x)-g(0)-g^{\prime \prime}(x)
$$

for $g$ locally absolutely continuous with $g \in L^{1}\left(e^{-|x|} d x\right), g^{\prime}$ also locally absolutely continuous and $g^{\prime \prime} \in L^{1}\left(e^{-|x|} d x\right)$. The operator $\mathcal{T}_{\text {Lap }, P R}$ is also discussed in [10] but not used in [10] because it did not fit in with Malliavin calculus as well as (2.5).

Even in the straightforward situation of a normal distribution, often a standardization is applied, as explained in the next example.

Example 2.4. For the standard normal distribution $\mathcal{N}(0,1)$ it is easy to write out the operator (2.1) explicitly to get $\mathcal{T}_{\mathcal{N}(0,1)}(f)(x)=f^{\prime}(x)-x f(x)$ acting on a wide class of functions $\mathcal{F}(\mathcal{N}(0,1))$ which includes all absolutely continuous functions with polynomial decay at $\pm \infty$. In particular the constant function 1 is in $\mathcal{F}(\mathcal{N}(0,1))$. A standardization of the form $f(x)=H_{n}(x) f_{0}(x)$ with $H_{n}$ the $n^{\text {th }}$ Hermite polynomial $\left(H_{0}(x)=1, H_{1}(x)=x, H_{2}(x)=x^{2}-1\right)$ gives as operator $\mathcal{A} f_{0}(x)=$ $H_{n}(x) f_{0}^{\prime}(x)-H_{n+1}(x) f_{0}(x)$, see for example [12].

It is also possible to study the behavior of functions $f_{h}$ under quite general conditions on $h$. For instance if $\mathcal{H}$ is the set of measurable functions $h: \mathbb{R} \rightarrow[0,1]$ (leading to the total variation measure) then $\mathcal{F}^{(1)}$ is contained in the collection of differentiable functions such that $\|f\| \leq \sqrt{\pi / 2}$ and $\left\|f^{\prime}\right\| \leq 2$; see for instance [19].

For the general normal distribution $\mathcal{N}\left(\mu, \sigma^{2}\right)$ the operator (2.1) gives

$$
\mathcal{T}_{\mathcal{N}\left(\mu, \sigma^{2}\right)}(f)(x)=f^{\prime}(x)-\frac{x-\mu}{\sigma^{2}} f(x) .
$$

The standardization $f(x)=\sigma^{2} g^{\prime}(x)$ yields the classical Ornstein-Uhlenbeck Stein operator $\mathcal{A} g(x)=$ $\sigma^{2} g^{\prime \prime}(x)-(x-\mu) g^{\prime}(x)$, see for example [2].

We call the passage from a parsimonious operator $\mathcal{T}_{P}$ (such as (2.4)) acting on the implicit class $\mathcal{F}(P)$ to a specific operator $\mathcal{A}_{P}$ (such as $(2.5)$ ) acting on a generic class $\mathcal{F}\left(\mathcal{A}_{P}\right)$ a standardization of $\left(\mathcal{T}_{P}, \mathcal{F}(P)\right)$. Given $P$ there are infinitely many different possible standardizations.

\subsection{The Stein transfer principle}

Suppose that we aim to assess the discrepancy between the laws of two random quantities $X$ with distribution $P$ and $W$ with distribution $Q$, say, in terms of some probability distance of the form

$$
d_{\mathcal{H}}(P, Q)=d_{\mathcal{H}}(X, W)=\sup _{h \in \mathcal{H}}|\mathbb{E}[h(W)]-\mathbb{E}[h(X)]|,
$$

for $\mathcal{H}$ some measure-determining class; many common distances can be written under the form (2.7), including the Kolmogorov distance (with $\mathcal{H}$ the collection of indicators of half lines), the 
Total Variation distance (with $\mathcal{H}$ the collection of indicators of Borel sets) and the 1-Wasserstein distance (see (1.1)). Here writing $d_{\mathcal{H}}(X, W)$ is a shorthand for (2.7): this distance is not random.

Let $P$ have Stein pair $\left(\mathcal{T}_{P}, \mathcal{F}(P)\right)$ and consider a standardization $\left(\mathcal{A}_{P}, \mathcal{F}\left(\mathcal{A}_{P}\right)\right)$ as described in Section 2.2. The first key idea in Stein's method is to relate the test functions $h$ of interest to a function $f=f_{h} \in \mathcal{F}\left(\mathcal{A}_{P}\right)$ through the so-called Stein equation

$$
h(x)-\mathbb{E}[h(X)]=\mathcal{A}_{P} f(x), \quad x \in \mathcal{I},
$$

so that, for $f_{h}$ solving (2.8), we get $h(W)-\mathbb{E}[h(X)]=\mathcal{A}_{P} f_{h}(W)$ and, in particular,

$$
\sup _{h \in \mathcal{H}}|\mathbb{E}[h(W)]-\mathbb{E}[h(X)]|=\sup _{f \in \mathcal{F}^{(1)}}\left|\mathbb{E}\left[\mathcal{A}_{P} f(W)\right]\right|
$$

where $\mathcal{F}^{(1)}=\mathcal{F}^{(1)}\left(\mathcal{A}_{P}, \mathcal{H}\right)=\left\{f \in \mathcal{F}\left(\mathcal{A}_{P}\right) \mid \mathcal{A}_{P} f=h-\mathbb{E}[h(X)]\right.$ for some $\left.h \in \mathcal{H}\right\}$. The first step in Stein's method thus consists in some form of transfer principle whereby one transforms the problem of bounding the distance $d_{\mathcal{H}}(P, Q)$ into that of bounding the expectations of the operators $\mathcal{A}_{P}$ over a specific class of functions.

Example 2.5. For the standard normal distribution, the operators $(2.1)$ and $(2.6)$ give $\mathcal{T}_{\mathcal{N}(0,1)}(f)(x)=$ $f^{\prime}(x)-x f(x)$. Bounding expressions of the form $\left|\mathbb{E}\left[f^{\prime}(W)-W f(W)\right]\right|$ as occurring in the r.h.s. of (2.9) is a potent starting point for Gaussian approximation problems. Prominent examples include $W=\sum_{i} \xi_{i}$ a standardized sum of weakly dependent variables, and $W=F(X)$ a functional of a Gaussian process; see e.g. [2, 23, 19] for an overview.

In general, the success of Stein's method for a particular target relies on the positive combination of three factors :

(i) the functions in $\mathcal{F}^{(1)}$ need to have "good" properties (e.g. be bounded with bounded derivatives),

(ii) the operator $\mathcal{A}_{P}$ needs to be amenable to computations (e.g. its expression should only involve polynomial functions),

(iii) there must be some "handle" on the expressions $\mathbb{E}\left[\mathcal{A}_{P} f(W)\right]$ (e.g. allowing for Taylor-type expansions or the application of couplings).

Conditions (i) to (iii) are satisfied for a great variety of target distributions (including the exponential, chi-squared, gamma, semi-circle, variance gamma and many others, see for example https://sites.google.com/site/yvikswan/about-stein-s-method for an up-to-date list).

\subsection{The Stein kernel}

One of the many keys to a successful application of Stein's method for a given target distribution $P$ lies in the properties of P's Stein kernel. We now review some properties of this quantity which will play a central role in our analysis; see [18] or [15] for details.

Definition 2.6. Let $P$ be a probability distribution with mean $\mu$, and let $X \sim P$. A Stein kernel of $P$ is a random variable $\tau_{P}(X)$ such that

$$
\mathbb{E}\left[\tau_{P}(X) \varphi^{\prime}(X)\right]=\mathbb{E}[(X-\mu) \varphi(X)]
$$

for all differentiable $\varphi: \mathbb{R} \rightarrow \mathbb{R}$ for which the expectation $\mathbb{E}[(X-\mu) \varphi(X)]$ exists.

The function $x \mapsto \tau_{P}(x)=\mathbb{E}\left[\tau_{P}(X) \mid X=x\right]$ is a Stein kernel (function) of $P$. If $P$ has interval support with closure $[a, b]$ then, letting $I d$ denote the identity function, it is not hard to see that

$$
\tau_{P}(x)=\mathcal{T}_{P}^{-1}(\mu-I d)(x)=\frac{1}{p(x)} \int_{a}^{x}(\mu-y) p(y) d y
$$

is the unique Stein kernel of $P$. Moreover the following properties of the Stein kernel are immediate consequences of its definition:

$$
\text { for all } x \in \mathbb{R} \text { we have that } \tau_{P}(x) \geq 0 \text { and } \mathbb{E}\left[\tau_{P}(X)\right]=\operatorname{Var}[X] \text {. }
$$


The Stein kernels for a wide variety of classical distributions (all members of the Pearson family, as it turns out) bear agreeable expressions; see [8, Table 1], [20, 21] or the forthcoming [7] for illustrations.

\subsection{Stein factors}

Let $P$ have a continuous density $p$ with mean $\mu$ and support $\mathcal{I}$ such that the closure of $\mathcal{I}$ is the interval $[a, b]$ (possibly with infinite endpoints). Let $\left(\mathcal{T}_{P}, \mathcal{F}(P)\right)$ be the Stein pair of $P$ and suppose that $P$ admits a Stein kernel $\tau_{P}(x)$, as described in Subsection 2.4. We introduce the standardized Stein pair $\left(\mathcal{A}_{P}, \mathcal{F}\left(\mathcal{A}_{P}\right)\right)$ with

$$
\mathcal{A}_{P} f(x)=\mathcal{T}_{P}\left(\tau_{P} f\right)(x)=\tau_{P}(x) f^{\prime}(x)+(\mu-x) f(x), \quad x \in \mathcal{I},
$$

and

$$
\begin{aligned}
& \mathcal{F}\left(\mathcal{A}_{P}\right)=\{f: \mathbb{R} \rightarrow \mathbb{R} \text { absolutely continuous such that } \\
& \qquad \lim _{x \rightarrow a} f(x) \int_{a}^{x}(\mu-u) p(u) d u=\lim _{x \rightarrow b} f(x) \int_{x}^{b}(\mu-u) p(u) d u=0 \\
& \left.\quad \text { and }\left(f(x) \int_{a}^{x}(\mu-u) p(u) d u\right)^{\prime} \in L^{1}(d x)\right\}
\end{aligned}
$$

Our next lemma shows that whenever applicable, standardization (2.12) satisfies requirement (i) from the end of Section 2.3.

Lemma 2.7. Let $\mathcal{H}=\operatorname{Lip}(1)$ be the collection of Lipschitz functions $h: \mathbb{R} \rightarrow \mathbb{R}$ with Lipschitz constant 1 and let $\mathcal{F}^{(1)}$ be the collection of $f \in \mathcal{F}\left(\mathcal{A}_{P}\right)$ such that $\mathcal{A}_{P} f=h-\mathbb{E}[h(X)]$ for some $h \in \mathcal{H}$. Then $\mathcal{F}^{(1)}$ is contained in the collection of functions $f$ such that $\|f\|_{\infty} \leq 1$.

Lemma 2.7 is strongly related to [5, Corollary 2.16], adapted to our framework. For the sake of completeness we present a proof of (a generalization of) this result at the end of the present paper. The key to our approach lies in the fact that the bound in Lemma 2.7 does not depend on the standardization of the target $P$; it is in particular independent of the mean and variance of $X \sim P$ or of any normalizing constant that might appear in the expression of the density of $P$.

\section{Comparing univariate continuous densities}

For $i=1,2$, let $P_{i}$ be a probability distribution with an absolutely continuous density $p_{i}(\cdot)$ having support $\mathcal{I}_{i}$ with closure $\overline{\mathcal{I}}_{i}=\left[a_{i}, b_{i}\right]$, for some $-\infty \leq a_{i}<b_{i} \leq+\infty$. Suppose that $\mathcal{I}_{2} \subset \mathcal{I}_{1}$ and define $\pi_{0}$ through

$$
p_{2}=\pi_{0} p_{1} .
$$

Associate with both distributions the Stein pairs $\left(\mathcal{T}_{i}, \mathcal{F}_{i}\right)$ for $i=1,2$, as well as the resulting construction from the previous section.

The product structure (3.1) implies a key connection between $\mathcal{T}_{1}$ and $\mathcal{T}_{2}$, namely

$$
\mathcal{T}_{2}(f)=\mathcal{T}_{1}(f)+f \frac{\pi_{0}^{\prime}}{\pi_{0}}=\mathcal{T}_{1}(f)+f\left(\log \pi_{0}\right)^{\prime}
$$

for all $f \in \mathcal{F}_{1} \cap \mathcal{F}_{2}$.

\subsection{Bounds on the Wasserstein distance between univariate continuous densities}

Our main objective in this section is to provide computable and meaningful bounds on the Wasserstein distance $d_{\mathcal{W}}\left(P_{1}, P_{2}\right)$, defined in $(1.1)$, in terms of $\pi_{0}$ and $P_{1}$, under the product structure (3.1). 
Theorem 3.1. For $i=1,2$, let $P_{i}$ be a probability distribution with an absolutely continuous density $p_{i}$ having support $\mathcal{I}_{i}$ with closure $\overline{\mathcal{I}}_{i}=\left[a_{i}, b_{i}\right]$, for some $-\infty \leq a_{i}<b_{i} \leq+\infty$; suppose that $\mathcal{I}_{2} \subset \mathcal{I}_{1}$ and let $X_{i} \sim P_{i}$ have finite means $\mu_{i}$ for $i=1,2$. Assume that $\pi_{0}=\frac{p_{2}}{p_{1}}$, defined on $\mathcal{I}_{2}$, is differentiable on $\mathcal{I}_{2}$, satisfies $\mathbb{E}\left|\left(X_{1}-\mu_{1}\right) \pi_{0}\left(X_{1}\right)\right|<\infty$ and

$$
\begin{gathered}
\left(\pi_{0}(x) \int_{a_{1}}^{x}\left(h(y)-\mathbb{E}\left[h\left(X_{1}\right)\right]\right) p_{1}(y) d y\right)^{\prime} \in L^{1}(d x) \\
\lim _{x \rightarrow a_{2}, b_{2}} \pi_{0}(x) \int_{a_{1}}^{x}\left(h(y)-\mathbb{E}\left[h\left(X_{1}\right)\right]\right) p_{1}(y) d y=0
\end{gathered}
$$

for all $h \in \mathcal{H}$, the set of Lipschitz-1 functions on $\mathbb{R}$. Then

$$
\left|\mathbb{E}\left[\pi_{0}^{\prime}\left(X_{1}\right) \tau_{1}\left(X_{1}\right)\right]\right| \leq d_{\mathcal{W}}\left(P_{1}, P_{2}\right) \leq \mathbb{E}\left[\left|\pi_{0}^{\prime}\left(X_{1}\right)\right| \tau_{1}\left(X_{1}\right)\right]
$$

where $\tau_{1}$ is the Stein kernel of $P_{1}$.

Proof. We first prove the lower bound. Let $X_{2} \sim P_{2}$. Start by noting that $d_{\mathcal{W}}\left(P_{1}, P_{2}\right) \geq \mid \mathbb{E}\left[X_{2}\right]-$ $\mathbb{E}\left[X_{1}\right] \mid$ because $I d \in \operatorname{Lip}(1)$. With (3.1) we get that

$$
\begin{aligned}
\mathbb{E}\left[X_{2}\right]-\mathbb{E}\left[X_{1}\right] & =\mathbb{E}\left[X_{1} \pi_{0}\left(X_{1}\right)\right]-\mu_{1} \\
& =\mathbb{E}\left[\left(X_{1}-\mu_{1}\right) \pi_{0}\left(X_{1}\right)\right] \\
& =\mathbb{E}\left[\tau_{1}\left(X_{1}\right) \pi_{0}^{\prime}\left(X_{1}\right)\right]
\end{aligned}
$$

where we used the fact that $\mathbb{E}\left[\pi_{0}\left(X_{1}\right)\right]=1$ and the definition $(2.10)$ of $\tau_{1}\left(X_{1}\right)$ in the last line.

Next we prove the upper bound. By the definition $(2.3), f_{h}=\mathcal{T}_{1}^{-1}\left(h-\mathbb{E}\left[h\left(X_{1}\right)\right]\right) \in \mathcal{F}_{1}$. On the other hand, Conditions (3.3) and (3.4) guarantee that $f_{h} \in \mathcal{F}_{2}$ for all $h$ because

$$
p_{2} f_{h}=\pi_{0}(x) \int_{a_{1}}^{x}\left(h(y)-\mathbb{E}\left[h\left(X_{1}\right)\right]\right) p_{1}(y) d y
$$

is necessarily absolutely continuous. We conclude that all functions $f_{h}=\mathcal{T}_{1}^{-1}\left(h-\mathbb{E}\left[h\left(X_{1}\right)\right]\right)$ belong to the intersection $\mathcal{F}_{1} \cap \mathcal{F}_{2}$. Hence

$$
\begin{aligned}
\mathbb{E}\left[h\left(X_{2}\right)\right]-\mathbb{E}\left[h\left(X_{1}\right)\right] & =\mathbb{E}\left[\mathcal{T}_{1}\left(f_{h}\right)\left(X_{2}\right)\right] \\
& =\mathbb{E}\left[\mathcal{T}_{1}\left(f_{h}\right)\left(X_{2}\right)\right]-\mathbb{E}\left[\mathcal{T}_{2}\left(f_{h}\right)\left(X_{2}\right)\right] \\
& =-\mathbb{E}\left[f_{h}\left(X_{2}\right)\left(\log \pi_{0}\right)^{\prime}\left(X_{2}\right)\right] .
\end{aligned}
$$

Equality (3.7) follows from the assumption that $f_{h} \in \mathcal{F}_{2}$ so that $\mathcal{T}_{2} f_{h}$ cancels when integrated with respect to $p_{2}$, whereas the last equality follows from Equation (3.2). Now we define $g_{h}=f_{h} / \tau_{1}$ and recall that $\tau_{1} \geq 0$ to get

$$
\left|\mathbb{E}\left[h\left(X_{2}\right)\right]-\mathbb{E}\left[h\left(X_{1}\right)\right]\right|=\left|\mathbb{E}\left[g_{h}\left(X_{2}\right)\left(\log \pi_{0}\right)^{\prime}\left(X_{2}\right) \tau_{1}\left(X_{2}\right)\right]\right| \leq\left\|g_{h}\right\|_{\infty} \mathbb{E}\left[\left|\left(\log \pi_{0}\right)^{\prime}\left(X_{2}\right)\right| \tau_{1}\left(X_{2}\right)\right] .
$$

It follows from Lemma 2.7 that $\left\|g_{h}\right\|_{\infty} \leq 1$ for all $h \in \operatorname{Lip}(1)$, yielding

$$
d_{\mathcal{W}}\left(P_{1}, P_{2}\right) \leq \mathbb{E}\left[\left|\left(\log \pi_{0}\right)^{\prime}\left(X_{2}\right)\right| \tau_{1}\left(X_{2}\right)\right]=\mathbb{E}\left[\left|\pi_{0}^{\prime}\left(X_{1}\right)\right| \tau_{1}\left(X_{1}\right)\right],
$$

the last equality again following from (3.1).

Assumptions (3.3) and (3.4) are crucial. While (3.4) is in a sense innocuous (because $\mathcal{I}_{2} \subset \mathcal{I}_{1}$ ), (3.3) is quite stringent yet hard to verify in practice. In Section 5 we provide a proof of the following explicit and easy to verify sufficient conditions on $p$ for these and hence Theorem 3.1 to hold.

Proposition 3.2. We use the notations of Theorem 3.1. Suppose that $\pi_{0}, p_{1}$ and $p_{2}$ are differentiable over their support and that their derivatives are integrable. Suppose that

$$
\lim _{x \rightarrow a_{2}, b_{2}} \pi_{0}(x) p_{1}(x) \tau_{1}(x)=\lim _{x \rightarrow a_{2}, b_{2}} p_{2}(x) \tau_{1}(x)=0 .
$$


Let $\rho_{1}=p_{1}^{\prime} / p_{1}$ and suppose also that

$$
\pi_{0}^{\prime} p_{1} \tau_{1}=p_{2}^{\prime} \tau_{1}-\rho_{1} \tau_{1} p_{2} \in L^{1}(d x) .
$$

Then Theorem 3.1 applies.

Example 3.3 (Distance between Gaussians). To compare two Gaussian distributions, $\mathcal{N}\left(\mu_{1}, \sigma_{1}^{2}\right)$ and $\mathcal{N}\left(\mu_{2}, \sigma_{2}^{2}\right)$, order them so that $\sigma_{2}^{2} \leq \sigma_{1}^{2}$, and if $\sigma_{1}=\sigma_{2}$ then assume that $\mu_{1}>\mu_{2}$. If $P_{1}$ is $\mathcal{N}\left(\mu_{1}, \sigma_{1}^{2}\right)$ then $\tau_{1}(x)=\sigma_{1}^{2}$ is constant (see e.g. [27]). With $P_{2}$ being $\mathcal{N}\left(\mu_{2}, \sigma_{2}^{2}\right)$, all conditions in Proposition 3.2 are satisfied. Applying Theorem 3.1 and noting that $\left(\log \pi_{0}(x)\right)^{\prime}=x\left(\frac{1}{\sigma_{1}^{2}}-\frac{1}{\sigma_{2}^{2}}\right)+$ $\left(\frac{\mu_{2}}{\sigma_{2}^{2}}-\frac{\mu_{1}}{\sigma_{1}^{2}}\right)$, we obtain that

$$
\begin{aligned}
\left|\mu_{2}-\mu_{1}\right| \leq d_{\mathcal{W}}\left(P_{1}, P_{2}\right) & \leq \sigma_{1}^{2} \mathbb{E}\left|X_{2}\left(\frac{1}{\sigma_{1}^{2}}-\frac{1}{\sigma_{2}^{2}}\right)+\left(\frac{\mu_{2}}{\sigma_{2}^{2}}-\frac{\mu_{1}}{\sigma_{1}^{2}}\right)\right| \\
& \leq\left|\frac{\sigma_{1}^{2}}{\sigma_{2}^{2}} \mu_{2}-\mu_{1}\right|+\left(\frac{\sigma_{1}^{2}}{\sigma_{2}^{2}}-1\right) \mathbb{E}\left|X_{2}\right| .
\end{aligned}
$$

In the special case $\mu_{2}=\mu_{1}=0$ we compute $\mathbb{E}\left|X_{2}\right|=\sqrt{2 / \pi} \sigma_{2}$ to get

$$
d_{\mathcal{W}}\left(P_{1}, P_{2}\right) \leq \sqrt{\frac{2}{\pi}} \frac{\sigma_{1}^{2}-\sigma_{2}^{2}}{\sigma_{2}},
$$

to be compared with a similar result in [19, Proposition 3.6.1].

If $\mu_{2} \neq 0$ then the general expression for $\mathbb{E}\left|X_{2}\right|$ is not agreeable, which is why we suggest using the inequality $\mathbb{E}\left|X_{2}\right| \leq\left(\mathbb{E}\left[X_{2}^{2}\right]\right)^{1 / 2}=\sqrt{\sigma_{2}^{2}+\mu_{2}^{2}}$, leading to

$$
\left|\mu_{2}-\mu_{1}\right| \leq d_{\mathcal{W}}\left(P_{1}, P_{2}\right) \leq\left|\frac{\sigma_{1}^{2}}{\sigma_{2}^{2}} \mu_{2}-\mu_{1}\right|+\left(\frac{\sigma_{1}^{2}}{\sigma_{2}^{2}}-1\right) \sqrt{\sigma_{2}^{2}+\mu_{2}^{2}} .
$$

With $\mu_{1}=\mu_{2}=\mu$, the upper bound becomes $\left(|\mu|+\sqrt{\sigma_{2}^{2}+\mu^{2}}\right)\left(\frac{\sigma_{1}^{2}}{\sigma_{2}^{2}}-1\right)$. We have not found a similar result in the literature (outside of the centered case) and computing the Wasserstein distance directly using (3.10) is prohibitive as the cdf's are not available in closed form.

Remark 3.4. Our upper bounds are not restricted to the Wasserstein case only. Indeed, mimicking large parts of the proof of Theorem 3.1, we obtain the general bound

$$
d_{\mathcal{H}}\left(P_{1}, P_{2}\right) \leq \kappa_{\mathcal{H}} \mathbb{E}\left[\left|\pi_{0}^{\prime}\left(X_{1}\right)\right| \tau_{1}\left(X_{1}\right)\right]
$$

with $\kappa_{\mathcal{H}}=\sup _{h \in \mathcal{H}}\left\|\mathcal{T}_{1}^{-1}\left(h-\mathbb{E}_{1} h\right) / \tau_{1}\right\|_{\infty}$ and $\mathcal{H}$ a measure-determining class of functions (the Kolmogorov distance corresponds to the class of indicators of half-lines, the Total Variation distance to the indicators of Borel sets). Usefulness of (3.8) hinges around availability of bounds similar to Lemma 2.7 on the more general constant $\kappa_{\mathcal{H}}$.

Unravelling the lower bound and using (2.11) in the upper bound of (3.5) we also obtain the following weaker but perhaps more transparent result.

Corollary 3.5. Under the same assumptions as for Theorem 3.1, with $X_{2} \sim P_{2}$,

$$
\left|\mathbb{E}\left[X_{2}\right]-\mathbb{E}\left[X_{1}\right]\right| \leq d_{\mathcal{W}}\left(P_{1}, P_{2}\right) \leq\left\|\pi_{0}^{\prime}\right\|_{\infty} \operatorname{Var}\left[X_{1}\right] .
$$

We shall use Corollary (3.9) in Section 4. We stress the fact that there is no normalizing constant appearing in the bounds (3.5) and (3.9). Also, the absence of Stein kernel in (3.9) is in somes cases an advantage because the Stein kernel is not always easy to compute.

There are many ways of expressing the Wasserstein distance (1.1) between two random variables. In general, if $P_{1}$ has cumulative distribution function (cdf) $F_{P_{1}}$ and if $P_{2}$ has cdf $F_{P_{2}}$ then

$$
d_{\mathcal{W}}\left(P_{1}, P_{2}\right)=\int_{\mathbb{R}}\left|F_{P_{1}}(x)-F_{P_{2}}(x)\right| d x=\int_{0}^{1}\left|F_{P_{1}}^{-1}(u)-F_{P_{2}}^{-1}(u)\right| d u=\inf \mathbb{E}\left|\xi_{1}-\xi_{2}\right|
$$


where the infimum in this last expression is taken over all possible couplings $\left(\xi_{1}, \xi_{2}\right)$ of $\left(P_{1}, P_{2}\right)$ (see e.g. [29, 30]). Often exact computable expressions of Wasserstein distances tend to be difficult to obtain. The similarity between the upper and lower bounds in (3.5) encourages us to formulate the next result.

Corollary 3.6. If $X_{i} \sim P_{i}, i=1,2$, are as in Theorem 3.1 and if $\pi_{0}$ is monotone increasing or decreasing, then

$$
d_{\mathcal{W}}\left(P_{1}, P_{2}\right)=\left|\mathbb{E}\left[X_{2}\right]-\mathbb{E}\left[X_{1}\right]\right|=\mathbb{E}\left[\left|\pi_{0}^{\prime}\left(X_{1}\right)\right| \tau_{1}\left(X_{1}\right)\right]=\mathbb{E}\left[\left|\left(\log \pi_{0}\right)^{\prime}\left(X_{2}\right)\right| \tau_{1}\left(X_{2}\right)\right] .
$$

Note how the second expression in (3.11) can be immediately obtained from the first by applying the same argument as in (3.6). Now while the second expression in (3.11) is new, the first is in fact not. Indeed the condition that $\pi_{0}$ be monotone in Corollary 3.6 is equivalent to requiring $X_{1} \geq_{L R} X_{2}$ (stochastically ordered in the sense of likelihood ratio, see e.g. [24, Section 9.4] or Example 3.8). If $X_{1} \leq_{L R} X_{2}$ then $F_{P_{2}} \leq F_{P_{1}}$ (see for example [25, Theorem 1.C.4]), so that $d_{\mathcal{W}}\left(X_{1}, X_{2}\right)=\int_{\mathbb{R}}\left(F_{P_{1}}(x)-F_{P_{2}}(x)\right) d x=\mathbb{E}\left[X_{2}\right]-\mathbb{E}\left[X_{1}\right]$.

Example 3.7 (Distance between Azzalini-type skew-symmetric distributions). Consider a symmetric density $p_{1}$ on the real line. The so-called Azzalini-type skew-symmetric distributions are constructed from such a pdf $p_{1}$ by considering the densities $p_{2}(x)=2 p_{1}(x) G(\lambda x)$ with $G$ the cdf of a univariate symmetric distribution with $p d f g$ and $\lambda \in \mathbb{R}$ a parameter (called skewness parameter); see [13] for an overview of these skewing mechanisms and of their applications. The founding example is Azzalini [1]'s skew-normal density $2 \phi(x) \Phi(\lambda x)$ (denoted $\mathcal{S} \mathcal{N}(0,1, \lambda)$ ), where $\phi$ and $\Phi$ respectively stand for the standard normal density and cumulative distribution function.

Corollary 3.6 provides, under mild conditions on $g$ and $G$, an exact expression for the Wasserstein distance between $P_{1}$ with $p d f p_{1}$ and its skew-symmetric counterpart $P_{2}$ with pdf $p_{2}$ since in this case $\left(\log \pi_{0}\right)^{\prime}(x)=\lambda g(\lambda x) / G(\lambda x)$ which is positive or negative depending on the sign of $\lambda$ as both $g$ and $G$ are positive on the support of $P_{2}$. Thus we have $\pi_{0}^{\prime}(x)=2 \lambda g(\lambda x)$ and

$$
d_{\mathcal{W}}\left(p_{1}, p_{2}\right)=2|\lambda| \mathbb{E}\left[\tau_{1}\left(X_{1}\right) g\left(\lambda X_{1}\right)\right] .
$$

Perhaps the most interesting instance of the above is the comparison of the standard normal with the skew-normal (all conditions in Proposition 3.2 are satisfied in this case) :

$$
d_{\mathcal{W}}(\mathcal{N}(0,1), \mathcal{S N}(0,1, \lambda))=\sqrt{\frac{2}{\pi}} \frac{|\lambda|}{\sqrt{1+\lambda^{2}}}
$$

(recall that $\tau_{1}(x)=1$ ). Letting $\lambda \rightarrow \infty$ we obtain that the distance between the half-normal with density $2 \phi(x) \mathcal{I}_{x \geq 0}$ and the normal is $\sqrt{2 / \pi}$, see also [6]. As in the previous example, such results are not easy to obtain directly from (3.10).

Likelihood ratio orderings have a natural role in comparing parametric densities. Let $p(x ; \theta)$ be a parametric family of densities with parameter of interest $\theta \in \mathbb{R}$ (see e.g. [17] for discussion and references). Set $p_{1}(\cdot)=p\left(\cdot ; \theta_{1}\right)$ and $p_{2}(\cdot)=p\left(\cdot ; \theta_{2}\right)$. The family $p(x ; \theta)$ is said to have monotone likelihood ratio if $x \mapsto p\left(x ; \theta_{2}\right) / p\left(x ; \theta_{1}\right)$ is non decreasing as soon as $\theta_{2}>\theta_{1}$ (and vice-versa). If $P_{1}$ has pdf $p_{1}$ and if $P_{2}$ has pdf $p_{2}$ then under monotone likelihood ratio, $P_{2} \leq P_{1}$. The property of monotone likelihood ratio is intrinsically linked with the validity of one-sided tests in statistics, see [14].

Example 3.8 (Distances within the exponential family). A noteworthy class of parametric distributions which satisfy the property of monotone likelihood ratio is the canonical regular exponential family $p(x ; \theta)=\ell(x) e^{\theta x-A(\theta)}$ for some scalar functions $\ell$ and $A$, with the range of the distribution being independent of $\theta$, see for example [14, page 639]. If $\theta_{1}>\theta_{2}$ then $\left(\log \pi_{0}\right)^{\prime}(x)=$ $\left(\log \frac{p_{2}(x)}{p_{1}(x)}\right)^{\prime}=\theta_{2}-\theta_{1}<0$ for all $x \in \mathbb{R}$ and thus from (3.11) we find with $X_{2} \sim P_{2}$ that $d_{\mathcal{W}}\left(P_{1}, P_{2}\right)=\left|\theta_{2}-\theta_{1}\right| \mathbb{E}\left[\tau_{1}\left(X_{2}\right)\right]$ under mild and easy-to-check conditions on $P_{1}$ and $P_{2}$. 
Example 3.9 (Distances between "tilted" distributions ). Fix a density $p_{1}$ with mean $\mu_{1}$ and consider, among all other densities $g$ with same support and fixed but different mean $\mu_{2} \neq \mu_{1}$, the density that minimizes the Kullback-Leibler divergence

$$
K L\left(g \| p_{1}\right)=\int g(x) \log \left(\frac{g(x)}{p_{1}(x)}\right) d x .
$$

The Euler-Lagrange equation for the constrained variational problem is $\log g(x)=\log p_{1}(x)+\lambda_{1} x+$ $\lambda_{2}$ solved by

$$
p_{2}(x)=p_{1}(x) \frac{e^{\lambda_{1} x}}{M_{1}\left(\lambda_{1}\right)}
$$

with $M_{1}(t)=\mathbb{E}\left[e^{t X_{1}}\right]$ the moment generating function of $X_{1} \sim p_{1}$ and $\lambda_{1}$ a solution to

$$
\frac{d}{d t}\left(\log M_{1}(t)\right)_{t=\lambda_{1}}=\mu_{2}
$$

in order to guarantee $\mathbb{E}\left[X_{2}\right]=\mu_{2}$. We call (3.12) a "tilted" version of $p_{1}$ (following the classical notion of exponential tilting, see e.g. [9]). It is easy to compute

$$
K L\left(p_{2} \| p_{1}\right)=\lambda_{1} \mu_{2}-\log M_{1}\left(\lambda_{1}\right) .
$$

Setting $\pi_{0}(x)=e^{\lambda_{1} x} / M_{1}\left(\lambda_{1}\right)$ we have $\log \left(\pi_{0}\right)^{\prime}(x)=\lambda_{1}$ and

$$
d_{\mathcal{W}}\left(p_{1}, p_{2}\right)=\left|\lambda_{1}\right| \mathbb{E}\left[\tau_{1}\left(X_{2}\right)\right]
$$

provided that the appropriate conditions are satisfied.

For the sake of illustration, take $p_{1}$ the Gamma distribution on the positive half line with density $p_{1}(x ; \lambda, k)=\frac{1}{\Gamma(k)} e^{-x / \lambda} x^{k-1} \lambda^{-k}$. Then $M_{1}(t)=(1-\lambda t)^{-k}$ for $t<\frac{1}{\lambda}$ and $\lambda_{1}=\frac{1}{\lambda}-\frac{k}{\mu_{2}}$. Moreover $\tau_{1}(x)=\lambda x$. It is thus easy to check in this case that all conditions in Proposition 3.2 are satisfied. This allows us to deduce from (3.13) that

$$
d_{\mathcal{W}}\left(p_{1}, p_{2}\right)=\left|\mu_{2}-\lambda k\right|
$$

which nicely complements $K L\left(p_{2} \| p_{1}\right)=\frac{\mu_{2}}{\lambda}-k+\log \left(\frac{k \lambda}{\mu_{2}}\right)^{k}$ as an alternative comparison statistic.

\section{On the influence of the prior in Bayesian statistics}

We now tackle the problem that motivated Theorem 3.1: assessing the impact of the choice of the prior distribution on the resulting posterior distribution in Bayesian statistics. In all examples we consider the conditions in Proposition 3.2 are easy to verify explicitly.

We first fix the notations. Assume that the observation $x$ comes from a parametric model with pdf $f(x ; \theta)$ with $\theta \in \Theta-f(x ; \theta)$ is often called the likelihood or the sampling density. We turn this model into a pdf for $\theta$ through

$$
p_{1}(\theta ; x)=\kappa_{1}(x) f(x ; \theta)
$$

where $\kappa_{1}(x)=\left(\int f(x ; \theta) d \theta\right)^{-1}$, and we assume that $\kappa_{1}<\infty$. Let $P_{1}$ have pdf $p_{1}$ and call its Stein kernel $\tau_{1}$. Choose a possibly improper prior density $\pi_{0}(\theta)$, and let

$$
p_{2}(\theta ; x)=\pi_{0}(\theta ; x) p_{1}(\theta ; x)
$$

where

$$
\pi_{0}(\theta ; x)=\kappa_{2}(x) \pi_{0}(\theta) \text { such that } \int p_{2}(\theta ; x) d \theta=1
$$

Then

$$
1=\int p_{2}(\theta ; x) d \theta=\kappa_{2}(x) \int \pi_{0}(\theta) p_{1}(\theta ; x) d \theta=\kappa_{2}(x) \mathbb{E}\left[\pi_{0}\left(\Theta_{1}\right)\right]
$$


where $\Theta_{1}$ has distribution $P_{1}$ which gives an expression for the normalizing constant. Let $P_{2}=$ $P_{2}(\cdot ; x)$ be the probability distribution on $\Theta$ with pdf $p_{2}(\cdot ; x)$. Then $P_{2}$ is the posterior distribution of $\theta$ under the prior $\pi_{0}$ and the data $x$; moreover $P_{1}$ can be seen as the distribution of $\theta$ under a uniform prior and the data $x$.

Now we extract from (3.5) of Theorem 3.1 the first bounds on the impact of a prior on the posterior distribution :

$$
\frac{\left|\mathbb{E}\left[\tau_{1}\left(\Theta_{1}\right) \pi_{0}^{\prime}\left(\Theta_{1}\right)\right]\right|}{\mathbb{E}\left[\pi_{0}\left(\Theta_{1}\right)\right]} \leq d_{\mathcal{W}}\left(P_{2}, P_{1}\right) \leq \frac{\mathbb{E}\left[\tau_{1}\left(\Theta_{1}\right)\left|\pi_{0}^{\prime}\left(\Theta_{1}\right)\right|\right]}{\mathbb{E}\left[\pi_{0}\left(\Theta_{1}\right)\right]}
$$

which can also be rewritten as

$$
\left|\mathbb{E}\left[\Theta_{2}\right]-\mathbb{E}\left[\Theta_{1}\right]\right|=\left|\mathbb{E}\left[\tau_{1}\left(\Theta_{2}\right) \rho_{0}\left(\Theta_{2}\right)\right]\right| \leq d_{\mathcal{W}}\left(P_{2}, P_{1}\right) \leq \mathbb{E}\left[\tau_{1}\left(\Theta_{2}\right)\left|\rho_{0}\left(\Theta_{2}\right)\right|\right]
$$

with $\Theta_{2} \sim P_{2}$ and

$$
\rho_{0}(\theta)=\frac{\pi_{0}^{\prime}(\theta)}{\pi_{0}(\theta)}
$$

the score function of $\pi_{0}(\theta ; x)$ with respect to $\theta$, which does not depend on the data $x$. As we shall see in the forthcoming sections which treat some classical examples in Bayesian statistics, (4.2) often turns out to be handier for computations than (4.1).

\subsection{A normal model}

Consider the simple setting where $x=\left(x_{1}, \ldots, x_{n}\right)$ is a random sample from a $\mathcal{N}\left(\theta, \sigma^{2}\right)$ population, where the scale $\sigma$ is known and the location $\theta$ is the parameter of interest, and assume that the prior $\pi_{0}(\theta)>0$ for all $\theta \in \Theta$ is differentiable. The likelihood $f(x ; \theta)$ of the normal model can be factorized into

$$
\begin{aligned}
f(x ; \theta) & =\left(2 \pi \sigma^{2}\right)^{-\frac{n}{2}} \exp \left\{-\frac{1}{2} \sum_{i=1}^{n} \frac{\left(x_{i}-\theta\right)^{2}}{\sigma^{2}}\right\} \\
& =\left(2 \pi \sigma^{2}\right)^{-\frac{n}{2}} \exp \left\{-\frac{1}{2 \sigma^{2}}\left(\sum_{i=1}^{n} x_{i}^{2}-n \bar{x}^{2}\right)\right\} \exp \left\{-\frac{1}{2} \frac{(\theta-\bar{x})^{2}}{\sigma^{2} / n}\right\} \\
& \propto \exp \left\{-\frac{1}{2} \frac{(\theta-\bar{x})^{2}}{\sigma^{2} / n}\right\} \text { when viewed as a function of } \theta
\end{aligned}
$$

where $\bar{x}=\frac{1}{n} \sum_{i=1}^{n} x_{i}$. Thus, $P_{1}=\mathcal{N}\left(\bar{x}, \sigma^{2} / n\right)$. Since $\tau_{1}$ is constant, equal to $\sigma^{2} / n$, the variance of $\Theta_{1} \sim P_{1}$, the bound (4.1) becomes

$$
\frac{\sigma^{2}}{n} \frac{\left|\mathbb{E}\left[\pi_{0}^{\prime}\left(\Theta_{1}\right)\right]\right|}{\mathbb{E}\left[\pi_{0}\left(\Theta_{1}\right)\right]} \leq d_{\mathcal{W}}\left(P_{2}, P_{1}\right) \leq \frac{\sigma^{2}}{n} \frac{\mathbb{E}\left[\left|\pi_{0}^{\prime}\left(\Theta_{1}\right)\right|\right]}{\mathbb{E}\left[\pi_{0}\left(\Theta_{1}\right)\right]}
$$

and (4.2) becomes

$$
\left|\mathbb{E}\left[\Theta_{2}\right]-\bar{x}\right|=\frac{\sigma^{2}}{n}\left|\mathbb{E}\left[\rho_{0}\left(\Theta_{2}\right)\right]\right| \leq d_{\mathcal{W}}\left(P_{1}, P_{2}\right) \leq \frac{\sigma^{2}}{n} \mathbb{E}\left[\left|\rho_{0}\left(\Theta_{2}\right)\right|\right] .
$$

Both inequalities are equalities in the case that $\pi_{0}$ is monotone.

\subsection{Normal prior and normal model}

Consider the same setting as in the previous section with the additional information that the prior $\pi_{0}$ is the density of a $\mathcal{N}\left(\mu, \delta^{2}\right)$, where $\mu$ and $\delta^{2}>0$ are known. Then the posterior $P_{2}$ is also normal, since

$$
p_{2}(\theta ; x) \propto \exp \left\{-\frac{1}{2}\left(\frac{(\theta-\bar{x})^{2}}{\sigma^{2} / n}+\frac{(\theta-\mu)^{2}}{\delta^{2}}\right)\right\} .
$$


Defining $a=\frac{n}{\sigma^{2}}+\frac{1}{\delta^{2}}$ and $b(x)=\frac{\bar{x}}{\sigma^{2} / n}+\frac{\mu}{\delta^{2}}$, we see that $P_{2}=\mathcal{N}\left(\frac{b(x)}{a}, \frac{1}{a}\right)$.

Since the prior $\pi_{0}$ is not monotone, we cannot exactly evaluate the Wasserstein distance between $P_{1}$ and $P_{2}$. However then we can write $\rho_{0}(\theta)=-(\theta-\mu) / \delta^{2}$ to obtain

$$
\frac{\sigma^{2}}{n \delta^{2}+\sigma^{2}}|\bar{x}-\mu| \leq d_{\mathcal{W}}\left(P_{1}, P_{2}\right) \leq \frac{\sigma^{2}}{n \delta^{2}+\sigma^{2}}|\bar{x}-\mu|+\frac{\sqrt{2}}{\sqrt{\pi}} \frac{\sigma^{3}}{n \delta \sqrt{\delta^{2} n+\sigma^{2}}} .
$$

To see this, the lower bound follows directly from simplifying the difference of the expectations,

$$
\left|\frac{b(x)}{a}-\bar{x}\right|=\frac{\sigma^{2}}{n \delta^{2}+\sigma^{2}}|\bar{x}-\mu| .
$$

For the upper bound, using $\rho_{0}(\theta)=-(\theta-\mu) / \delta^{2}$ in (4.3) gives

$$
\begin{aligned}
& d_{\mathcal{W}}\left(P_{1}, P_{2}\right) \leq \frac{\sigma^{2}}{n} \mathbb{E}\left[\left|\rho_{0}\left(\Theta_{2}\right)\right|\right] \\
& =\frac{\sigma^{2}}{n \delta^{2}} \mathbb{E}\left[\left|\Theta_{2}-\mu\right|\right] \\
& \leq \frac{\sigma^{2}}{n \delta^{2}}\left(\mathbb{E}\left[\left|\Theta_{2}-\frac{b(x)}{a}\right|\right]+\left|\frac{b(x)}{a}-\mu\right|\right) \\
& =\frac{\sqrt{2}}{\sqrt{\pi}} \sqrt{\frac{1}{a}} \frac{\sigma^{2}}{n \delta^{2}}+\frac{\sigma^{2}}{n \delta^{2}}\left|\frac{b(x)}{a}-\mu\right| \\
& =\frac{\sqrt{2}}{\sqrt{\pi}} \frac{\delta \sigma}{\sqrt{\delta^{2} n+\sigma^{2}}} \frac{\sigma^{2}}{n \delta^{2}}+\frac{\sigma^{2}}{n \delta^{2}} \frac{\delta^{2}}{\delta^{2}+\frac{\sigma^{2}}{n}}|\bar{x}-\mu| \\
& =\frac{\sqrt{2}}{\sqrt{\pi}} \frac{\sigma^{3}}{n \delta \sqrt{\delta^{2} n+\sigma^{2}}}+\frac{\sigma^{2}}{n \delta^{2}+\sigma^{2}}|\bar{x}-\mu|,
\end{aligned}
$$

which yields the upper bound in (4.4).

Inequality (4.4) provides a quite concrete and intuitive idea of the impact of the prior. First we see that, for $n \rightarrow \infty$, the distance becomes zero, as is well known. The prior variance $\delta^{2}$ has the same influence, which is also natural given that the prior then tends towards an improper prior, too. If the data are unfavourable so that $|\bar{x}-\mu|$ is large compared to $n$, then the Wasserstein distance between the two posterior distributions will be large. Due to the law of large numbers, for large $n$ the probability that $|\bar{x}-\mu|>\delta^{2} n+\sigma^{2}$ is small; but in contrast to such asymptotic considerations, the bound (4.4) makes the influence of the data on the distance explicit. Further the upper and lower bounds only differ by an $O\left(n^{-3 / 2}\right)$ term, hence at a $1 / n$ precision, we have an exact expression for the Wasserstein distance. Finally, the $O(1 / n)$ term in both bounds perfectly reflects the intuition that the better the guess of the prior mean $\mu$ (w.r.t. the data), the smaller the influence of the prior.

\subsection{The binomial model}

As next example we treat the case of $n$ independent and identically distributed Bernoulli random variables with parameter of interest $\theta \in[0,1]$; alternatively, we may say we have a single observation $y \in\{0,1, \ldots, n\}$ from a Binomial distribution with known $n$ and parameter of interest $\theta$. The corresponding sampling density is

$$
f(y ; \theta)=\left(\begin{array}{l}
n \\
y
\end{array}\right) \theta^{y}(1-\theta)^{n-y}
$$

and $p_{1}(\theta ; y)=\kappa_{1}(y) \theta^{y}(1-\theta)^{n-y}$ is a Beta density with

$$
\kappa_{1}(y)=\frac{1}{B(y+1, n-y+1)},
$$


where $B(\cdot, \cdot)$ denotes the Beta function, and $P_{1}=P_{1}(\cdot ; y)=\operatorname{Beta}(y+1, n-y+1)$ is a Beta distribution.

Recall that, if $X \sim p(x)=\frac{1}{B(\alpha, \beta)} x^{\alpha-1}(1-x)^{\beta-1}$ then

$$
\mathbb{E}[X]=\frac{\alpha}{\alpha+\beta}, \mathbb{E}\left[X^{2}\right]=\frac{\alpha(1+\alpha)}{(\alpha+\beta)(\alpha+\beta+1)} \text { and } \operatorname{Var}[X]=\frac{\alpha \beta}{(\alpha+\beta)^{2}(\alpha+\beta+1)} .
$$

The Stein kernel is $\tau(x)=\frac{x(1-x)}{\alpha+\beta}$ and in particular $\tau_{1}(\theta)=\frac{\theta(1-\theta)}{n+2}$. Corollary 3.5 gives that, for any differentiable prior $\pi_{0}$ on $\mathcal{I}=[0,1]$,

$$
d_{\mathcal{W}}\left(P_{1}, P_{2}\right) \leq \sup _{0 \leq \theta \leq 1}\left|\pi_{0}^{\prime}(\theta)\right| \frac{(y+1)(n-y+1)}{(n+2)^{2}(n+3)} .
$$

For $y$ close to $\frac{n}{2}$, this bound is of order $n^{-1}$. In particular, for any $0 \leq y \leq n$, for a prior with bounded derivative, the Wasserstein distance converges to zero as $n \rightarrow \infty$ no matter which data are observed, but the data may affect the rate of convergence. Next we consider some choices of prior densities which may not have bounded derivatives.

\subsubsection{Beta prior}

For a Beta prior

$$
\pi_{0}(\theta) \propto \theta^{\alpha-1}(1-\theta)^{\beta-1},
$$

the assumptions of Theorem 3.1 are satisfied but $\sup _{0 \leq \theta \leq 1}\left|\pi_{0}^{\prime}(\theta)\right|$ is infinite unless both $\alpha$ and $\beta$ are greater than or equal to 2 (or $\alpha=\beta=1$ ). Let $P_{1}$ denote the $\operatorname{Beta}(y+1, n-y+1)$ distribution and $P_{2}$ the posterior distribution using the prior (4.5). It is well known that $P_{2}$ is again Beta distributed : the Beta distributions are conjugate priors for the Binomial distribution (similarly as the normal prior is conjugate in the normal model, see the previous section); in fact, it is easy to see that $P_{2}$ is the $\operatorname{Beta}(\alpha+y, \beta+n-y)$ distribution.

We shall show that

$$
\begin{aligned}
\left|\frac{y+1}{n+2}\left(\frac{\alpha+\beta-2}{n+\alpha+\beta}\right)-\frac{\alpha-1}{n+\alpha+\beta}\right| & \leq d_{\mathcal{W}}\left(P_{1}, P_{2}\right) \\
& \leq \frac{1}{n+2}\left\{|\alpha-1|+\frac{y+\alpha}{n+\alpha+\beta}(|\beta-1|-|\alpha-1|)\right\}
\end{aligned}
$$

To this end, let $\Theta_{1} \sim P_{1}$ and $\Theta_{2} \sim P_{2}$. With (4.2) we have the immediate lower bound on the Wasserstein distance, namely

$$
\begin{aligned}
d_{\mathcal{W}}\left(P_{1}, P_{2}\right) & \geq\left|\mathbb{E}\left[\Theta_{2}\right]-\mathbb{E}\left[\Theta_{1}\right]\right| \\
& =\left|\frac{y+1}{n+2}-\frac{y+\alpha}{n+\alpha+\beta}\right| \\
& =\left|\frac{y+1}{n+2}\left(1-\frac{n+2}{n+\alpha+\beta}\right)-\frac{\alpha-1}{n+\alpha+\beta}\right| \\
& =\left|\frac{y+1}{n+2}\left(\frac{\alpha+\beta-2}{n+\alpha+\beta}\right)-\frac{\alpha-1}{n+\alpha+\beta}\right| .
\end{aligned}
$$

For an upper bound, we calculate that

$$
\rho_{0}(\theta)=\frac{(\alpha-1)(1-\theta)-(\beta-1) \theta}{\theta(1-\theta)}
$$

and hence

$$
\tau_{1}(\theta) \rho_{0}(\theta)=\frac{1}{n+2}\{(\alpha-1)(1-\theta)-(\beta-1) \theta\}
$$


Using (4.2) we obtain the claimed upper bound

$$
\begin{aligned}
d_{\mathcal{W}}\left(P_{1}, P_{2}\right) & \leq \frac{1}{n+2} \mathbb{E}\left|(\alpha-1)\left(1-\Theta_{2}\right)-(\beta-1) \Theta_{2}\right| \\
& \leq \frac{1}{n+2}\left\{|\alpha-1| \mathbb{E}\left[1-\Theta_{2}\right]+|\beta-1| \mathbb{E}\left[\Theta_{2}\right]\right\} \\
& =\frac{1}{n+2}\left\{|\alpha-1|+\frac{y+\alpha}{n+\alpha+\beta}(|\beta-1|-|\alpha-1|\} .\right.
\end{aligned}
$$

Some comments on the bound (4.6) are in order. Firstly, both the upper and the lower bound vanish when $\alpha=\beta=1$. Secondly, unless $\alpha=1$, the upper bound is of order $O\left(n^{-1}\right)$, no matter how favourable the data $y$ are.

\subsubsection{The Jeffreys prior}

An alternative popular prior is

$$
\pi_{0}(\theta)=\frac{1}{\sqrt{\theta(1-\theta)}},
$$

the so-called Jeffreys prior obtained for $\alpha=\beta=1 / 2$ in (4.5). This is an improper prior which satisfies the assumptions of Theorem 3.1. The posterior distribution $P_{2}$ is $\operatorname{Beta}\left(y+\frac{1}{2}, n-y+\frac{1}{2}\right)$. Moreover

$$
\rho_{0}(\theta)=\frac{2 \theta-1}{2 \theta(1-\theta)}
$$

and

$$
\tau_{1}(\theta) \rho_{0}(\theta)=\frac{1}{2(n+2)}(2 \theta-1)
$$

Using (4.2) we obtain that

$$
\frac{1}{(n+1)}\left|\frac{y+1}{n+2}-\frac{1}{2}\right| \leq d_{\mathcal{W}}\left(P_{1}, P_{2}\right)
$$

and

$$
d_{\mathcal{W}}\left(P_{1}, P_{2}\right) \leq \frac{1}{n+2}\left\{\sqrt{\frac{\left(y+\frac{1}{2}\right)\left(n-y+\frac{1}{2}\right)}{(n+2)(n+1)^{2}}}+\left|\frac{y+\frac{1}{2}}{n+1}-\frac{1}{2}\right|\right\}
$$

The upper bound follows from the Cauchy-Schwarz inequality via

$$
\begin{aligned}
d_{\mathcal{W}}\left(P_{1}, P_{2}\right) & \leq \frac{1}{2(n+2)} \mathbb{E}\left|\left(2 \Theta_{2}-1\right)\right| \\
& \leq \frac{1}{n+2}\left\{\mathbb{E}\left|\Theta_{2}-\mathbb{E}\left[\Theta_{2}\right]\right|+\left|\mathbb{E}\left[\Theta_{2}\right]-\frac{1}{2}\right|\right\} \\
& \leq \frac{1}{n+2}\left\{\sqrt{\operatorname{Var}\left[\Theta_{2}\right]}+\left|\mathbb{E}\left[\Theta_{2}\right]-\frac{1}{2}\right|\right\} \\
& =\frac{1}{n+2}\left\{\sqrt{\frac{\left(y+\frac{1}{2}\right)\left(n-y+\frac{1}{2}\right)}{(n+2)(n+1)^{2}}}+\left|\frac{y+\frac{1}{2}}{n+1}-\frac{1}{2}\right|\right\} .
\end{aligned}
$$

In contrast to (4.6), the Jeffreys prior can achieve a bound of order $O\left(n^{-\frac{3}{2}}\right)$ if the data $y$ is close to $\frac{n}{2}$. 


\subsection{A Poisson model}

The last case we tackle is the Poisson model with data $x=\left(x_{1}, \ldots, x_{n}\right)$ from a Poisson distribution with sampling density

$$
f(x ; \theta)=e^{-n \theta} \frac{\theta^{\sum_{i=1}^{n} x_{i}}}{\prod_{i=1}^{n} x_{i} !} .
$$

When $\sum_{i=1}^{n} x_{i} \neq 0$, which we shall now assume, then we obtain that $P_{1}$, the posterior distribution under a uniform prior, has pdf

$$
p_{1}(\theta ; x) \propto \exp (-\theta n) \theta^{\sum_{i=1}^{n} x_{i}+1-1}
$$

a gamma density with parameters $1 / n$ and $\sum_{i=1}^{n} x_{i}+1$; its Stein kernel is simply $\tau_{1}(\theta)=\theta / n$ (see Example 3.9). The general bound (3.9) from Corollary 3.5 becomes

$$
d_{\mathcal{W}}\left(P_{1}, P_{2}\right) \leq \sup _{\theta \geq 0}\left|\pi_{0}^{\prime}\left(\theta ; \sum x_{i}\right)\right| \frac{\bar{x}+\frac{1}{n}}{n},
$$

where $\bar{x}=\frac{1}{n} \sum_{i=1}^{n} x_{i} \geq \frac{1}{n}$.

Taking for $\theta$ a negative exponential prior $\operatorname{Exp}(\lambda)$ with $\lambda>0$,

$$
\pi_{0}(\theta)=\lambda e^{-\lambda \theta}
$$

over $\mathbb{R}^{+}$yields that the posterior $P_{2}$ has density $p_{2}(\theta ; x) \propto \exp (-\theta(n+\lambda)) \theta^{\sum_{i=1}^{n} x_{i}+1-1}$, again a gamma density where the first parameter is updated to $1 /(n+\lambda)$. Here, the prior is monotone decreasing, hence we can exactly calculate the effect of the prior to obtain

$$
\begin{aligned}
d_{\mathcal{W}}\left(P_{1}, P_{2}\right) & \left.=\mathbb{E}\left[\mid \log \pi_{0}\left(\Theta_{2}\right)\right)^{\prime} \mid \frac{\Theta_{2}}{n}\right] \\
& =\lambda \frac{\mathbb{E}\left[\Theta_{2}\right]}{n} \\
& =\lambda \frac{\bar{x}+\frac{1}{n}}{n+\lambda} \\
& =\frac{\lambda}{n+\lambda} \bar{x}+\frac{\lambda}{n(n+\lambda)} .
\end{aligned}
$$

We note that the exact distance differs from the general bound (4.7) here only through a multiplicative factor $\frac{n}{\lambda(n+\lambda)}\left(\right.$ since $\left.\sup _{\theta \geq 0}\left|\pi_{0}^{\prime}\left(\theta ; \sum x_{i}\right)\right|=\lambda^{2}\right)$. The distance increases with $\bar{x}$ but will always be at least as large as $\frac{\lambda}{n(n+\lambda)}$. As we assume that $\bar{x} \geq \frac{1}{n}$, the data-dependent part of the Wasserstein distance will always be at least as large as the part which stems solely from the prior. Finally, from the strong law of large numbers, $\bar{x}$ will almost surely converge to a constant as $n \rightarrow \infty$, so that the Wasserstein distance will converge to 0 almost surely.

\section{Technical results}

In this section we first prove the variant of Corollary 2.16 of [5] which we use in our paper. It includes Lemma 2.7 as a special case.

Lemma 5.1. Let $P$ have a continuous density $p$ with mean $\mu$ and support $\mathcal{I}$ an interval with closure $\overline{\mathcal{I}}=[a, b]$ with $-\infty \leq a<b \leq+\infty$ and let $X \sim P$. Write $F_{P}$ for the corresponding cumulative distribution function. Let $h: \mathcal{I} \rightarrow \mathbb{R}$ be Lebesgue-almost surely differentiable such that the Fubini condition

$$
\int_{A} \int_{B}\left|h^{\prime}(v)\right| p(u) d v d u=\int_{B} \int_{A}\left|h^{\prime}(v)\right| p(u) d u d v<\infty
$$

is satisfied for all Borel-measurable subsets $A, B \subset[a, b]$. Then 
1 .

$$
\left|\int_{a}^{x}(h(y)-\mathbb{E}[h(X)]) p(y) d y\right| \leq\left\|h^{\prime}\right\| \int_{a}^{x}(\mu-y) p(y) d y ;
$$

2. for $g_{h}=\frac{\mathcal{T}_{P}^{-1}(h-\mathbb{E}[h(X)])}{\tau_{P}}$ it holds that

$$
\left\|g_{h}\right\| \leq\left\|h^{\prime}\right\|
$$

3. [Lemma 2.7] in particular, if $\mathcal{H}$ is the set of all Lipschitz-continuous functions $h: \mathcal{I} \rightarrow \mathbb{R}$ with Lipschitz constant 1, then

$$
\left\|g_{h}\right\| \leq 1
$$

for all $h \in \mathcal{H}$.

Proof. We prove the three items separately, closely following [5] and in particular his Lemma 5.3.

1. Let $h: \mathcal{I} \rightarrow \mathbb{R}$ be as detailed in the assumptions. Then, under the sole assumption that Fubini is allowed, we can write for all $a \leq y \leq b$

$$
\begin{aligned}
h(y)-\mathbb{E}[h(X)] & =\int_{a}^{b}(h(y)-h(u)) p(u) d u \\
& =\int_{a}^{b} \int_{u}^{y} h^{\prime}(v) p(u) d v d u \\
& =\int_{a}^{y} \int_{u}^{y} h^{\prime}(v) p(u) d v d u-\int_{y}^{b} \int_{y}^{u} h^{\prime}(v) p(u) d v d u \\
& =\int_{a}^{y} \int_{a}^{v} h^{\prime}(v) p(u) d u d v-\int_{y}^{b} \int_{v}^{b} h^{\prime}(v) p(u) d u d v \\
& =\int_{a}^{y} F_{P}(v) h^{\prime}(v) d v-\int_{y}^{b}\left(1-F_{P}(v)\right) h^{\prime}(v) d v .
\end{aligned}
$$

Integrating the above w.r.t. $p$ and again applying Fubini we get after straightforward simplifications

$$
\begin{aligned}
& \int_{a}^{x}(h(y)-\mathbb{E}[h(X)]) p(y) d y \\
& =-\left(1-F_{P}(x)\right) \int_{a}^{x} F_{P}(s) h^{\prime}(s) d s-F_{P}(x) \int_{x}^{b}\left(1-F_{P}(s)\right) h^{\prime}(s) d s
\end{aligned}
$$

for each $x \in[a, b]$ from which we readily derive

$$
\begin{aligned}
& \left|\int_{a}^{x}(h(y)-\mathbb{E}[h(X)]) p(y) d y\right| \\
& \leq\left\|h^{\prime}\right\|\left(\left(1-F_{P}(x)\right) \int_{a}^{x} F_{P}(s) d s+F_{P}(x) \int_{x}^{b}\left(1-F_{P}(s)\right) d s\right) .
\end{aligned}
$$

To deal with this last expression we use the identities

$$
\int_{a}^{x} F_{P}(s) d s=x F_{P}(x)-\int_{a}^{x} s p(s) d s
$$

and

$$
\int_{x}^{b}\left(1-F_{P}(s)\right) d s=-x\left(1-F_{P}(x)\right)+\int_{x}^{b} s p(s) d s .
$$

Straightforward computations yield the claim. 
2. For Item 2, by definition

$$
\mathcal{T}_{P}^{-1}(h(x)-\mathbb{E}[h(X)])=\frac{1}{p(x)} \int_{a}^{x}(h(y)-\mathbb{E}[h(X)]) p(y) d y .
$$

Also, by definition,

$$
\tau_{P}(x) p(x)=\int_{a}^{x}(\mu-y) p(y) d y
$$

Hence

$$
g_{h}(x)=\frac{\int_{a}^{x}(h(y)-\mathbb{E}[h(X)]) p(y) d y}{\int_{a}^{x}(\mu-y) p(y) d y}
$$

which, by Item 1 , satisfies

$$
\left\|g_{h}\right\| \leq\left\|h^{\prime}\right\|\left|\frac{\int_{a}^{x}(\mu-y) p(y) d y}{\int_{a}^{x}(\mu-y) p(y) d y}\right|=\left\|h^{\prime}\right\| .
$$

3. Item 3 follows directly from Rademacher's Theorem for Lipschitz functions which guarantees that they are almost surely differentiable, with derivative bounded by 1 if their Lipschitz constant is 1 .

We conclude the paper with a proof of Proposition 3.2, restated for convenience.

Proposition 5.2. We use the notations of Theorem 3.1. Suppose that $\pi_{0}, p_{1}$ and $p_{2}$ are differentiable over their support and that their derivatives are integrable. Suppose that

$$
\lim _{x \rightarrow a_{2}, b_{2}} \pi_{0}(x) p_{1}(x) \tau_{1}(x)=\lim _{x \rightarrow a_{2}, b_{2}} p_{2}(x) \tau_{1}(x)=0 .
$$

Let $\rho_{1}=p_{1}^{\prime} / p_{1}$ and suppose also that

$$
\pi_{0}^{\prime} p_{1} \tau_{1}=p_{2}^{\prime} \tau_{1}-\rho_{1} \tau_{1} p_{2} \in L^{1}(d x)
$$

Then Theorem 3.1 applies.

Proof. Conditions (3.3) and (3.4) are equivalent to requiring that $f_{h} \in \mathcal{F}_{2}$, in other words $\left(f_{h} p_{2}\right)$ needs to be differentiable, $\left(f_{h} p_{2}\right)^{\prime}$ needs to be integrable with integral on $\mathcal{I}_{2}$ (the support of $p_{2}$ ) equal to 0 . By definition,

$$
f_{h}(x) p_{2}(x)=\pi_{0}(x) \int_{a_{1}}^{x}\left(h(y)-\mathbb{E}\left[h\left(X_{1}\right)\right]\right) p_{1}(y) d y
$$

is differentiable if $\pi_{0}$ is differentiable. Next, differentiating,

$$
\left(f_{h} p_{2}\right)^{\prime}(x)=\pi_{0}^{\prime}(x) \int_{a_{1}}^{x}\left(h(y)-\mathbb{E}\left[h\left(X_{1}\right)\right]\right) p_{1}(y) d y+\pi_{0}(x)\left(h(x)-\mathbb{E}\left[h\left(X_{1}\right)\right]\right) p_{1}(x) .
$$

For the second summand, the Lipschitz property of $h$ gives the bound

$$
\left|h(x)-\mathbb{E}\left[h\left(X_{1}\right)\right]\right| \leq \int_{a_{1}}^{b_{1}}|h(x)-h(y)| p_{1}(y) d y \leq \int_{a_{1}}^{b_{1}}|x-y| p_{1}(y) d y,
$$

so that

$$
\int_{a_{1}}^{b_{1}}\left|\pi_{0}(x)\left(h(x)-\mathbb{E}\left[h\left(X_{1}\right)\right]\right) p_{1}(x)\right| d x \leq \int_{a_{1}}^{b_{1}} p_{2}(x) \int_{a_{1}}^{b_{1}}|x-y| p_{1}(y) d y d x \leq \mathbb{E}\left|X_{1}\right|+\mathbb{E}\left|X_{2}\right|,
$$


and the latter expectations are assumed to exist. Hence in order to guarantee (3.3) it is sufficient to impose that

$$
\pi_{0}^{\prime}(x) \int_{a_{1}}^{x}\left(h(y)-\mathbb{E}\left[h\left(X_{1}\right)\right]\right) p_{1}(y) d y \in L^{1}(d x) .
$$

We can write

$$
\int_{a_{1}}^{x}\left(h(y)-\mathbb{E}\left[h\left(X_{1}\right)\right]\right) p_{1}(y) d y=p_{1}(x) \tau_{1}(x) g_{h}(x)
$$

with

$$
g_{h}(x)=\frac{1}{\tau_{1}(x) p_{1}(x)} \int_{a_{1}}^{x}\left(h(y)-\mathbb{E}\left[h\left(X_{1}\right)\right]\right) p_{1}(y) d y
$$

a function which we know from Lemma 5.1 to be bounded uniformly by 1 . Hence (5.1) (and therefore (3.3)) boils down to a condition on $\pi_{0}^{\prime}(x) p_{1}(x) \tau_{1}(x)$. Similarly (3.4) can be tracked down to a condition on $\pi_{0}(x) p_{1}(x) \tau_{1}(x)$, and the claim follows.

\section{References}

[1] A. Azzalini. A class of distributions which includes the normal ones. Scandinavian Journal of Statistics, 12:171-178, 1985.

[2] L. H. Y. Chen, L. Goldstein, and Q.-M. Shao. Normal Approximation by Stein's Method. Probability and its Applications (New York). Springer, Heidelberg, 2011.

[3] P. Diaconis and D. Freedman. On inconsistent Bayes estimates of location. The Annals of Statistics, 14(1):68-87, 1986.

[4] P. Diaconis and D. Freedman. On the consistency of Bayes estimates. The Annals of Statistics, $14(1): 1-67,1986$. With a discussion and a rejoinder by the authors.

[5] C. Döbler. Stein's method of exchangeable pairs for absolutely continuous, univariate distributions with applications to the Pólya urn model. Preprint arXiv:120\%.0533, 2012.

[6] C. Döbler. Stein's method for the half-normal distribution with applications to limit theorems related to simple random walk. arXiv preprint arXiv:1303.4592, 2013.

[7] C. Döbler, R. E. Gaunt, C. Ley, G. Reinert, and Y. Swan. A handbook of Stein operators. In preparation, 2014.

[8] R. Eden and J. Viquez. Nourdin-Peccati analysis on Wiener and Wiener-Poisson space for general distributions. Stochastic Processes and Their Applications, 125:182-216, 2015.

[9] B. Efron. Nonparametric standard errors and confidence intervals. The Canadian Journal of Statistics/La Revue Canadienne de Statistique, 9: 139-158, 1981.

[10] P. Eichelsbacher and C. Thäle. Malliavin-Stein method for Variance-Gamma approximation on Wiener space. arXiv preprint arXiv:1409.5646, 2014.

[11] R. E. Gaunt. Variance-Gamma approximation via Stein's method. Electronic Journal of Probability, 19(38):1-33, 2014.

[12] L. Goldstein and G. Reinert. Distributional transformations, orthogonal polynomials, and Stein characterizations. Journal of Theoretical Probability 18: 237-260, 2005.

[13] M. Hallin and C. Ley. Skew-symmetric distributions and Fisher information: the double sin of the skew-normal. Bernoulli, 20(3):1432-1453, 2014.

[14] S. Karlin and H. Rubin. Distributions possessing a monotone likelihood ratio. Journal of the American Statistical Association, 51(276): 637-643, 1956.

[15] C. Ley, G. Reinert, and Y. Swan. Approximate computation of expectations: a canonical Stein operator. arXiv preprint arXiv:1408.2998, 2014.

[16] C. Ley and Y. Swan. Local Pinsker inequalities via Stein's discrete density approach. IEEE Transactions on Information Theory, 59(9):5584-4491, 2013.

[17] C. Ley and Y. Swan. Parametric Stein operators and variance bounds. Brazilian Journal of Probability and Statistics, 2015.

[18] C. Ley and Y. Swan. Stein's density approach and information inequalities. Electronic Communications in Probability, 18(7):1-14, 2013. 
[19] I. Nourdin and G. Peccati. Normal Approximations with Malliavin Calculus : from Stein's Method to Universality. Cambridge Tracts in Mathematics. Cambridge University Press, 2012.

[20] I. Nourdin, G. Peccati, and Y. Swan. Entropy and the fourth moment phenomenon. Journal of Functional Analysis, 266:3170-3207, 2014.

[21] I. Nourdin, G. Peccati, and Y. Swan. Integration by parts and representation of information functionals. 2014 IEEE International Symposium on Information Theory (ISIT), 2217-2221, 2014.

[22] J. Pike and H. Ren. Stein's method and the Laplace distribution. ALEA, 11: 571-587, 2014.

[23] N. Ross. Fundamentals of Stein's method. Probability Surveys, 8:210-293, 2011.

[24] S. M. Ross. Stochastic Processes, volume 2. John Wiley \& Sons New York, 1996.

[25] M. Shaked and J. G. Shanthikumar. Stochastic Orders. Springer Science \& Business Media, 2007.

[26] C. Stein. Approximation of improper prior measures by prior probability measures. In Bernoulli 1713, Bayes 1763, Laplace 1813, pages 217-240. Springer, 1965.

[27] C. Stein. Approximate Computation of Expectations. Institute of Mathematical Statistics Lecture Notes-Monograph Series, 7. Institute of Mathematical Statistics, Hayward, CA, 1986.

[28] C. Stein, P. Diaconis, S. Holmes, and G. Reinert. Use of exchangeable pairs in the analysis of simulations. In P. Diaconis and S. Holmes, editors, Stein's Method: Expository Lectures and Applications, volume 46 of IMS Lecture Notes Monogr. Ser., pages 1-26. Beachwood, Ohio, USA: Institute of Mathematical Statistics, 2004.

[29] S. Vallender. Calculation of the Wasserstein distance between probability distributions on the line. Theory of Probability and Its Applications, 18(4):784-786, 1974.

[30] C. Villani. Optimal Transport: Old and New, volume 338. Springer Science \& Business Media, 2008. 